\title{
physiokongress
}

25.-27. Januar 2013

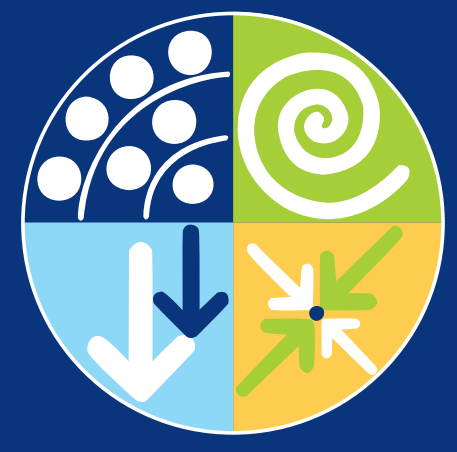

\section{Ein voller Erfolg}

PHYSIOKONGRES 2013 Rund 600 Physiotherapeuten trafen sich zum siebten physiokongress und der ersten TheraPro. Ein Highlight war die Session „Hunt the Pain!“, bei dem die Therapeuten Roland Gautschi und Dr. Claus Beyerlein sowie der Osteopath Frank Roels einen ihnen vorher unbekannten Patienten befundeten und anschließend therapierten. Welchen Eindruck das Kongress-Wochenende sonst noch bei den Besuchern hinterlassen hat, fragte physiopraxis nach.

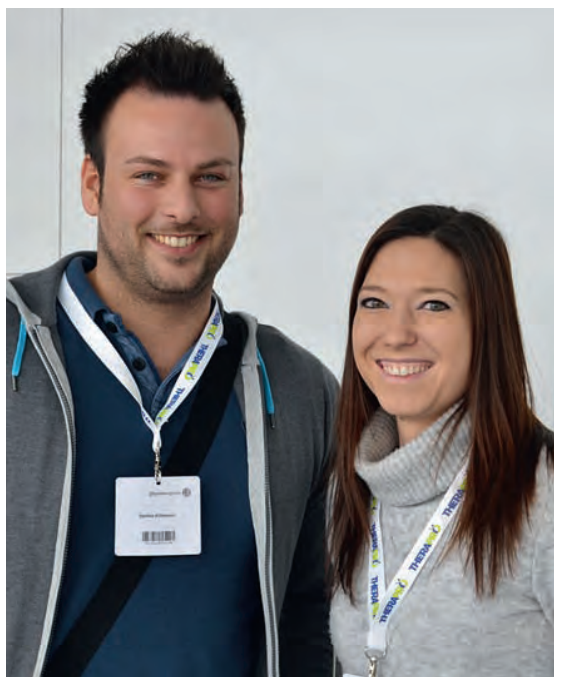

Steffen Kittmann und Uli Linhart

Den Vortrag „Physiotherapie bei unspezifischen Rückenschmerzen: Wir brauchen alle Muskeln“ von Prof. Hannu Luomajoki fanden wir angenehm zum Zuhören. Er war sehr aktuell und relevant für den Praxisalltag. „Unser Geist! Mentale Stärke" hat uns hingegen nicht so gefallen, da das Race Across America aus Sicht eines Mentaltrainers uninteressant für uns war. Aus physiotherapeutischer Sicht wäre der Vortrag sicherlich interessanter gewesen.
Cornelia Wiese, Dorothee Comes und Susanne Dohme Uns haben Vitalität und Elan von Susanna Freivogel gefallen. Ihr Thema „Was ist Spastik?" war hochinteressant, aber auch schwer. Beim Vortrag „Spastik in der Therapie nutzen oder hemmen?" hätte der praktische Bezug deutlicher sein können. Das HirnstimulationsThema war uns zu weit weg von dem, was wir machen. Das Therapiemanagement bei subluxierter Schulter war sehr gut, sehr praxisorientiert. Doch alles in allem hat uns der Neuroreha-Teil leider enttäuscht. Auch „Unser Geist! Mentale Stärke“ war nichts für uns. Das fiel stark nach dem sehr guten Vortrag von Prof. Dieter Felsenberg ab.



Maureen Müller

Bei „Hunt the Pain!" war sehr schön zu sehen, dass viele Wege nach Rom führen und manchmal der einfachste direkt zum Ziel führt. 
Lisa-Marie Gärtner (r.)

„Hunt the Pain!“ war für mich sehr informativ, da man gesehen hat, wie ein Problem auf unterschiedliche Art und Weise, also in unterschiedlichen Fachrichtungen, untersucht und behandelt werden kann.

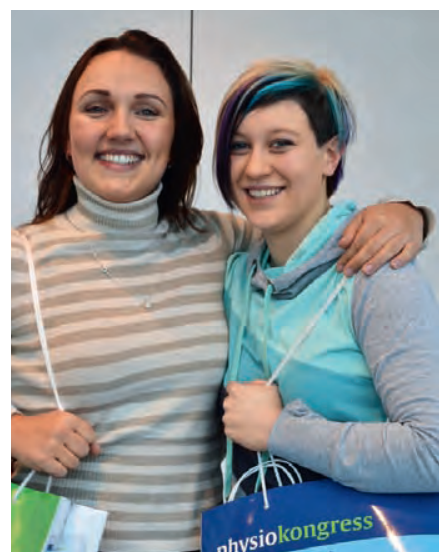

Marlene Zweng (I.)

Bei „Hunt the Pain!" verging die Zeit sehr schnell, da alles locker und praktisch gestaltet war und das Publikum super einbezogen wurde.

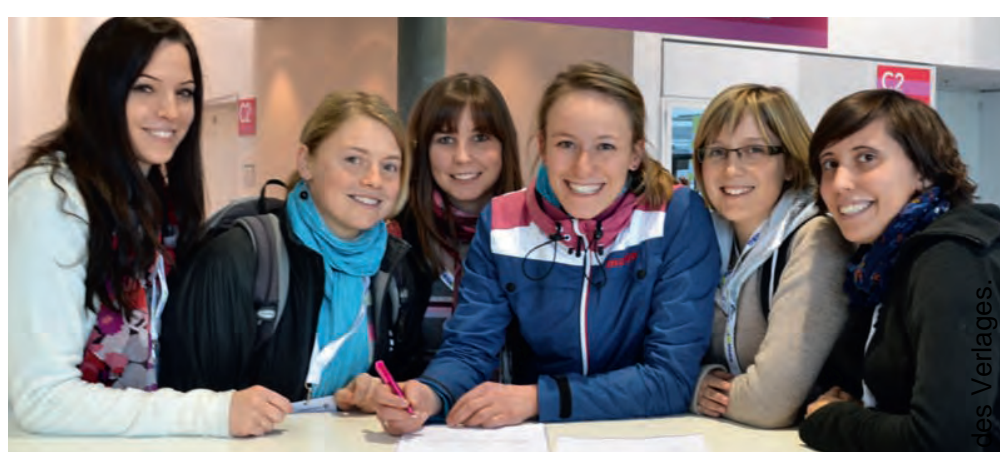

Angehende Physiotherapeutinnen von der Physiotherapieschule in Günzburg

Für uns setzte der Vortrag „Was ist Spastik?“ zu viel Wissen voraus. Die Geschwindigkeit war zu schnell, um gut folgen und das Gehörte reflektieren zu können. Der Vortrag von Martin Huber gefiel uns besser und verschaffte noch einmal einen Gesamtüberblick über das komplexe Thema Spastik. Das Thema „Hirnstimulationsverfahren und ihre Wirkung auf den Muskeltonus“ war zwar interessant, aber im Moment ist es für uns in der Anwendung in der Praxis nicht relevant.

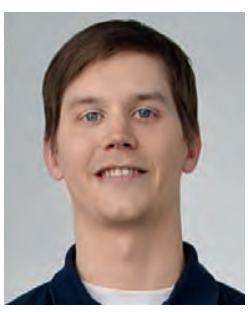

René Lehwalder

Der Vortrag „Unser Geist! Mentale Stärke“ war sehr interessant, weil man erfahren hat, wie wichtig die mentale Kraft und die Ziele im Leben sind, um gesund zu sein beziehungsweise zu bleiben.

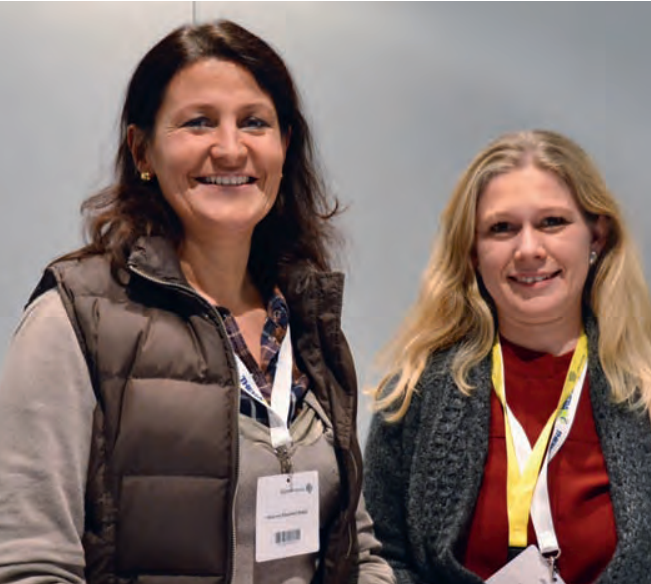

Anna von Eisenhart Rothe (I.)

Schön war, dass es bei „Hunt the Pain!“ nicht darum ging, wer recht hat und die Lösung findet. Jeder Zuhörer konnte sich in einem der drei unterschiedlichen Therapeuten-Typen wiederfinden. Authentische Vorträge, die den hohen Stellenwert der exakten Anamnese verdeutlichten.

Tanja Bossmann (r.)

Bei „Hunt the Pain!“ war es schön zu sehen, dass es trotz unterschiedlicher Konzepte doch auch viele Gemeinsamkeiten in Anamnese, Untersuchung und Behandlung gab. Toll war auch der wertschätzende und respektvolle Umgang untereinander.
Daniela Cisnovschi (I.)

Den Trånen mah und die Augen in persönlicher und therapeutischer Hinsicht. Ich kann mich für den Vortrag ,Unser Geist! Mentale Stårke ${ }^{66}$ nur bedanken.

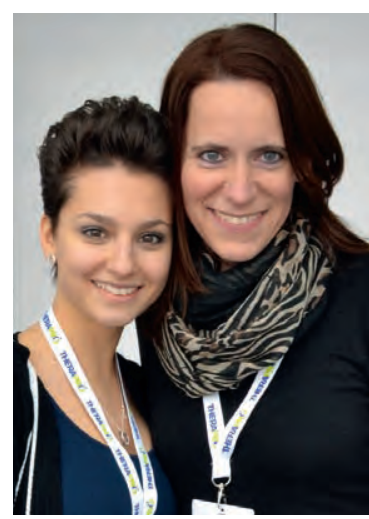

Dagmar Spittler (r.)

Die Eröffnungsvorträge waren überwältigend. Die Referenten verstanden es, die Zuhörer zu fesseln und aufmerksam zu machen auf neue Denkweisen in der Physiotherapie. 
Ingrid Opitz-Roser

Es war eine ganz neue Erfahrung, wie in „Hunt the Pain!“vor Ort ein Patient vorgestellt wurde. Auch span-

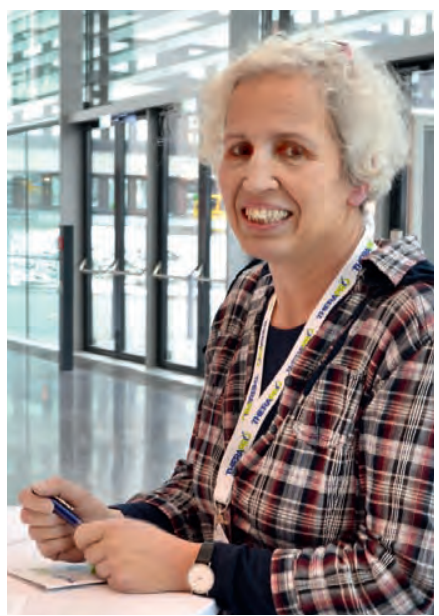
nend, wie die Therapeuten vorgingen und wie das Publikum voll einbezogen wurde.

\section{Kristine Müller-Lagaly und Maren Baudisch}

Uns hat „Hunt the Pain!“ sehr gut gefallen, da man seine eigene Art zu befunden reflektieren konnte. Die Vorträge zu „Muskelgeführter Schulterkomplex“ mit

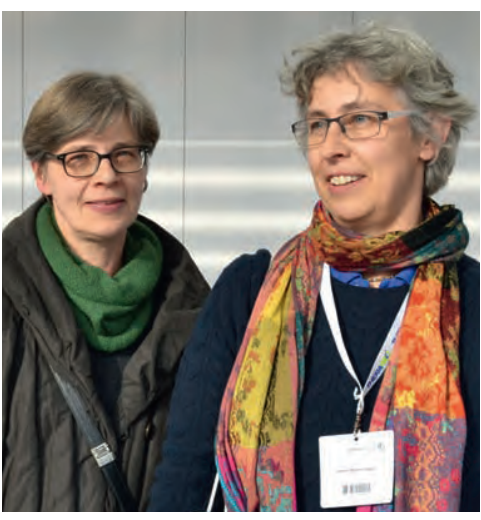

tollen Videos und Bildern waren ein super Update für die Schulter. Klasse war auch, dass Prof. Gerhard Bauer „pro KG“ eingestellt war. Von der TheraPro sind wir allerdings enttäuscht, da nur wenig physiotherapeutische Aussteller da sind.

PHYSIOKONGRESS 2014 VORMERIKEN

\section{Immer am letzten} Januar-Wochenende

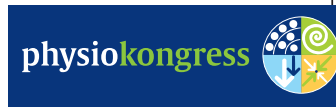

Wer im nächsten Jahr (wieder) beim physiokongress dabei sein möchte, kann sich jetzt schon mal das Wochenende vom 24. bis 26. Januar 2014 im Kalender vormerken. Beim physiokongress werden wieder ein muskuloskeletales Thema sowie die Neuroreha im Mittelpunkt stehen. Für die zweite TheraPro würden sich die Kongressbesucher deutlich mehr Aussteller mit Physio- und Ergotherapieangeboten wünschen. 